\title{
Short-term Distributed Energy Resource Scheduling for a DC Microgrid
}

\author{
G. W. Chang, H. J. Lu, H. J. Su \\ Department of Electrical Engineering, National Chung Cheng University \\ Email: wchang@ee.ccu.edu.tw \\ Received September, 2012
}

\begin{abstract}
A microgrid is associated with a low voltage distribution power network and inherits small modular generation systems and loads that have certain coordinated functions to provide the solution to supply premium power to remote or specific areas. Similar to conventional power systems, the energy management of distributed generation resources (DERs) is carried out to minimize the operation cost and maximize benefit of installation of DERS in a microgrid. This paper presents the process of implementing the short-term DER scheduling function for a dc microgrid. The optimal scheduling results for two operation modes are then reported.
\end{abstract}

Keywords: Microgrid; Distributed Generation Resources; PV; Battery

\section{Introduction}

The microgrid is a group of traditional and/or distributed generation resources, energy storages, and loads that is normally connected to a low-voltage power network. The point of common coupling (PCC) with the connected power network can be disconnected and the microgrid is then functioned in islanding mode. The microgrid can provide the platform for integration of different renewable resources with communications [1].

Due to characteristics of distributed energy resources, modern power systems have functions of changing its power flow either in one direction or bi-directions. Since it is a trend that active distributed systems have flexible and smart control systems to obtain clean energy from renewable sources, distribution systems have transformed into larger-scale of smarter grids or a smaller scale of localized microgrids. Microgrids can reduce cost of investment for installations of renewable sources and they also provide reactive power compensation and frequency regulation, increase capacity of spinning reserve, and improve power quality and reliability of customer's power system.

Unlike the conventional power system, a microgrid may include energy-storage devices such as batteries and becomes a strong coupling system in the time domain [2-4]. Furthermore, due to uncontrollable characteristics of DERs, certain operation function such as islanding mode of the grid and have to be further studied. In this paper, the short-term DER scheduling that considers the photovoltaic cell, wind generation, fuel cell, and energy- storage device (i.e. battery) of a dc microgrid supplying local loads is presented.

The optimization model is developed for the considered short-term DER scheduling problem. The objective is to minimize operational costs while satisfying the microgrid load demand. All the DER and energy-storage units must be operated within their lower and upper output limits. The output of the power optimization model considers the operation limits of the supply options, load demand, sell/purchase power costs from the connected grid, and the operating costs of the DERs. Therefore, the objective function becomes minimizing operational costs represented by the difference between the profit and the cost. In the scheduling model, the problem is formulated as a nonlinear programming problem is solved by an optimization toolbox provided by Matlab. To solve the microgrid DER scheduling problem, a solution algorithm is proposed and implemented under MATLAB Graphical User Interface Development Environment (GUIDE). Results are then presented to show the usefulness of the proposed algorithm.

\section{Problem Formulation}

Figure 1 shows the network configuration of the dc microgrid under study, where the DERs in the system include a wind turbine with the permanent magnet synchronous generator (PMSG), PV arrays, and a fuel cell. The energy-storage system is a battery. Two types of loads are in the system: one is the DC load fed by the DC bus and the other is the $\mathrm{AC}$ load connected to the $\mathrm{AC}$ 
grid. Interconnection between the DC microgrid and the $\mathrm{AC}$ grid is through a bi-directional inverter. To ensure a stable operation, the power generation and consumption must be balanced to keep the DC bus voltage within an acceptable range. The inverter and battery are responsible for DC voltage regulation in the grid-connected and islanding modes, respectively. In the grid-connected mode, the battery acts like a constant current source and can be in charge, discharge, or non-operated mode. In the islanding mode, the inverter is mainly responsible for maintaining a stable voltage to the $\mathrm{AC}$ loads.

\subsection{Photovoltaic Array Model}

The single-diode equivalent PV model shown in Figure 2 describes the v-i characteristic of PV arrays. In this model, the series resistance $\left(R_{S}\right)$ and the parallel resistance $\left(R_{P}\right)$ represent the effect PV conversion efficiency [5]. The equation describing the $\mathrm{v}-\mathrm{i}$ characteristic is

$$
i=i_{P H}-i_{0}\left[\exp \left(\frac{q\left(v+i R_{s}\right)}{k T A}\right)-1\right]-\frac{v+i R_{s}}{R_{P}}
$$

where $i$ is the output current of the PV, $i_{P H}$ is the current generated by the incident light, $i_{0}$ is the reverse saturation or the leakage current of diodes, $q$ is the electron charge, $v$ is the output voltage of the PV, $k$ is Boltzmann constant,

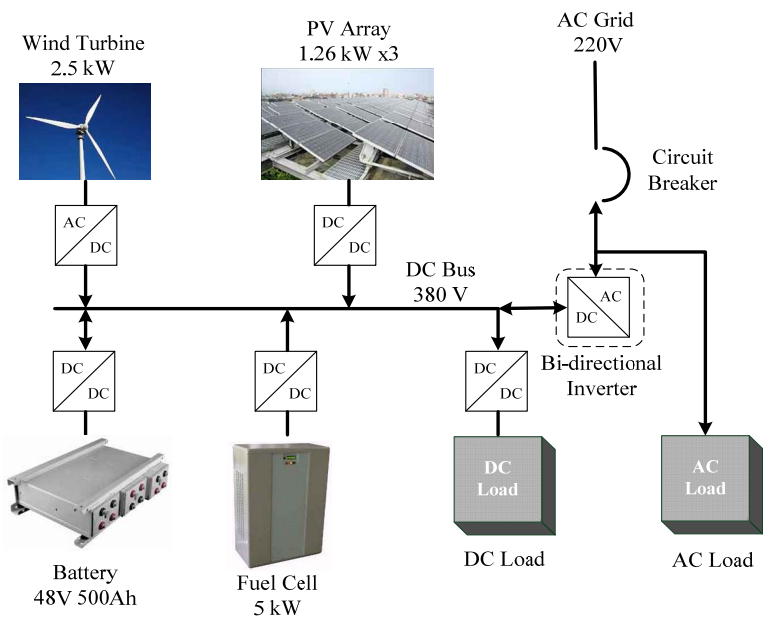

Figure 1. A typical configuration of DC microgrid.

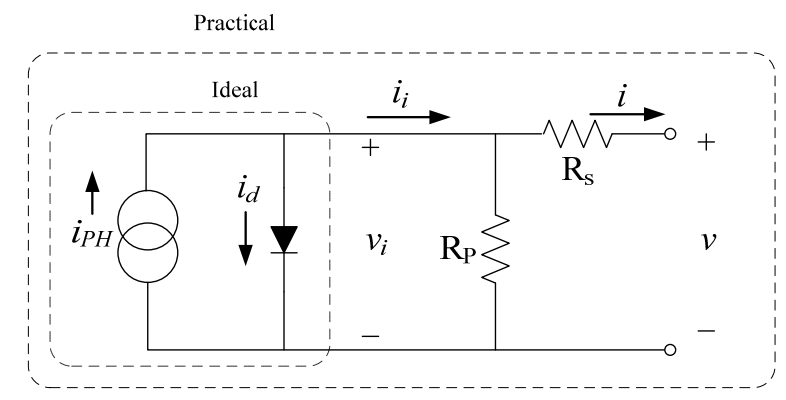

Figure 2. Equivalent single-diode PV mode.
$T$ is the temperature in Kelvin, $A$ is the ideal factor of the $\mathrm{PV}(A=1 \sim 1.5), R_{P}$ is the series resistance, and $R_{S}$ is the parallel resistance. The parameters needed in the equivalent PV model are $i_{P H}, i_{0}, R_{P}, R_{S}$, and $A$; they can be obtained from the datasheets and the procedure for calculating both series and parallel resistances $[6,7]$.

\subsection{Wind Turbine Model}

To model the permanent magnet synchronous generator (PMSG) of the wind turbine, the parameters of the PMSG are required to establish the detailed wind turbine models, which include the numbers of poles, dq-axis inductances, rotor resistance, etc. However, these parameters are usually not provided by manufactories, the measurement-based wind turbine models can be obtained by establishing a look-up table of wind speed versus output power characteristic curve to represent wind turbine power characteristic [8].

\subsection{Models of Fuel Cell and Battery}

The fuel cell stack model shown in Figure 3 [9] is commonly used and the parameters can be obtained from the datasheet shown in Figure 4, where $E_{o c}$ is the open circuit voltage, $i_{0}$ is the exchange current, $A$ is the Tafel slope, $N$ is the number of cells, and $T_{d}$ is the response

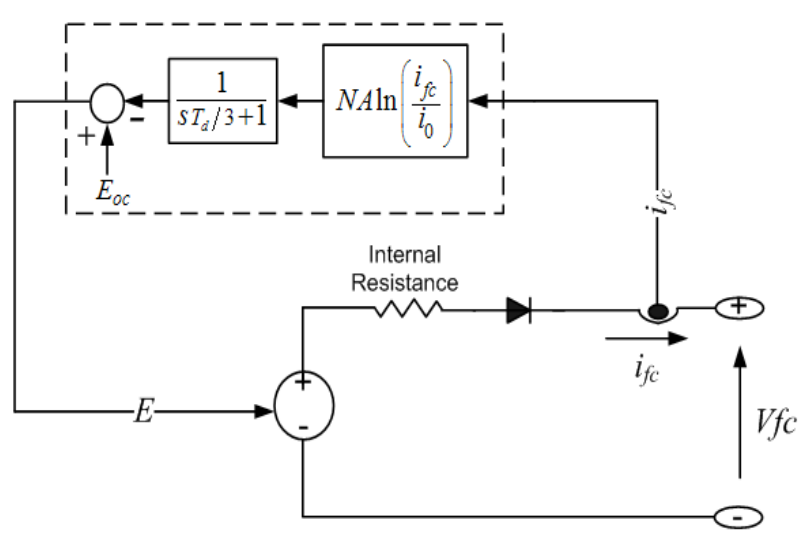

Figure 3. A commonly used model for the fuel cell [9].

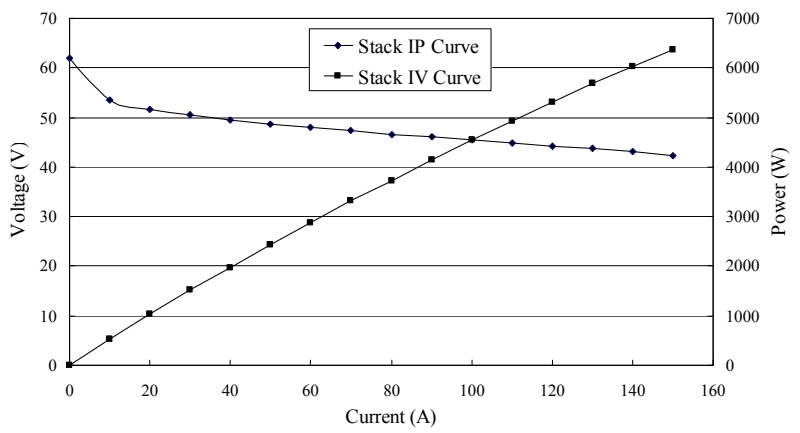

Figure 4. Current-power (i-p) and current-voltage (i-v) characteristic curves of a 5-kW fuel cell. 
time. The simplified model is based on the equivalent circuit of a fuel cell stack and represents a particular fuel cell stack operating at nominal conditions of temperature and pressure. Battery models in SPS include four predefined types: lead-acid, lithium ion, nickel metal hydride and nickel cadmium. Parameters of models are battery types, nominal voltage, rated capacity and initial charge state.

\section{Short-term DER Scheduling of the DC Microgrid}

\subsection{Objective Function and Constraints}

The short-term DER scheduling of the dc microgrid is to achieve its minimum operation cost. If the microgrid includes $N$ controllable DERs over a study period of $T$ time steps, the objective function to be minimized under grid-connected and islanding modes can be expressed by (2) and (3), respectively.

$$
\begin{gathered}
C=\sum_{t=1}^{T}\left[\left(\sum_{n=1}^{N} F_{n}\left(P_{n, t}\right)\right)+F_{\text {grid }}\left(P_{\text {grid }, t}\right)+F_{\text {batt }}\left(P_{\text {bat }, t}\right)\right] \\
C=\sum_{t=1}^{T}\left[\left(\sum_{n=1}^{N} F_{n}\left(P_{n, t}\right)\right)+F_{\text {batt }}\left(P_{\text {batt }, t}\right)\right]
\end{gathered}
$$

where $P_{n, t}$ is the output power of the $n^{\text {th }}$ DER in the $t$-th time step. $P_{\text {grid,t }}$ and $P_{\text {batt }, t}$ are the output power of the connected grid (i.e. electric utility) and the energy-storage device, respectively. $F_{n}, F_{\text {grid }}$, and $F_{\text {batt }}$ are cost functions associated with the $n^{\text {th }}$ DER, power sell or purchase of the electric utility, and energy-storage device, respectively. The constraints must be met at each time step are listed in (4)-(8).

$$
\begin{gathered}
\sum_{n=1}^{N} P_{n, t}+P_{\text {grid }, t}+P_{\text {batt }, t}=P_{\text {load }, t}-P_{\text {unctrl }, t} \\
P_{n}^{\min } \leq P_{n, t} \leq P_{n}^{\max } \\
P_{\text {grid }}^{\min } \leq P_{\text {grid }, t} \leq P_{\text {grid }}^{\max } \\
-P_{\text {charge }}^{\min } \leq P_{\text {batt }, t} \leq P_{\text {discharge }}^{\max } \\
\text { SOC }_{\text {min }} \leq S O C_{t-1}+\Delta S O C_{t} \leq S O C_{\text {max }}
\end{gathered}
$$

In (4)-(8), (4) is the power balance requirement during the $t$-th time step, which ensures a stable operation. $P_{\text {unctrl, } t}$ is output power of uncontrollable DERs (i.e., wind generation and PV arrays) during the $t$-th time step, and $P_{\text {load, } t}$ is total active power of dc and ac loads; (5) is generation limits for the $n$-th DER, where $P_{n}^{\min }$ and $P_{n}^{\max }$ are minimum and maximum generation, respectively. Equation (6) is inequality constrain of power from utility, and $P_{\text {grid }}^{\min }$ and $P_{\text {grid }}^{\max }$ are minimum and maximum power limitation. Equation (7) is associated with the charging and discharging power limits of the battery. In (8), the state-of-charge (SOC) of the battery must meet its normal operation constraints, where $S O C_{\min }$ and $S O C_{\max }$ are the lower and upper energy storage limits for the battery. $\Delta S O C_{t}$ is the change of SOC during the $t$-th time step.

\subsection{Proposed Solution Procedure for the DER Scheduling Problem}

To solve the described short-term DER scheduling problem for the micro grid in both islanding and grid-connected modes, it is assumed that, at each time step, the forecasts of power generation from uncontrollable DERs (i.e. PV and wind generator), the utility electricity price, the load consumption, the initial value of battery SOC and the microgrid operation mode are provided. In the grid-connected mode, the scheduling problem of (2)-(7) will be solved and the battery output and the power purchase from the connected utility grid at each time step will be determined at the minimum microgrid operation cost. In the islanding mode, the microgrid is disconnected form the utility grid. Consequently, the problem is without considering the $P_{\text {gird }}$ terms in the objective function and constraints and is then solved. For both operation modes, the scheduling problems are solved by the Matlab Optimization toolbox (quadratic programming solver) implemented with MATLAB GUIDE. Figure 5 illustrates the flowchart of the proposed solution procedure for both microgrid operation modes.

\section{Case Study}

In this study, the simulations for an actual dc microgrid with grid-connected and islanding modes for 48 time steps (each time step spans over 15 minutes) are performed to show the scheduling results. The associated cost functions and constraints are listed in (9)-(17).

$$
\begin{gathered}
F_{F C}\left(P_{F C}\right)=0.77 P_{F C}^{2}-4.94 P_{F C}+19.36 \\
0.5 k W \leq P_{F C} \leq 5 k W \\
F_{\text {grid_in }}\left(P_{\text {grid }}\right)=3 P_{\text {grid }} \\
F_{\text {grid_out }}\left(P_{\text {grid }}\right)=5 P_{\text {grid }} \\
-5 k W \leq P_{\text {grid }} \leq 5 \mathrm{~kW} \\
F_{\text {batt_charge }}\left(P_{\text {batt }}\right)=-1.5 P_{\text {batt }} \\
F_{\text {batt_discharge }}\left(P_{\text {batt }}\right)=1.6 P_{\text {batt }} \\
-10 k W \leq P_{\text {batt }} \leq 10 \mathrm{~kW} \\
85 \% \leq S O C_{t-1}+\Delta S O C_{t} \leq 90 \%
\end{gathered}
$$

where (9) and (10) are the fuel cell cost function and associated generation limits. In (11)-(16), positive value of $P_{\text {grid }}$ and $P_{\text {batt }}$ implies that the electric power flows into the microgrid. 


\subsection{Case 1: Grid-Connected Mode}

Figure 6 shows the forecasting curves of load demands and uncontrollable DERs for input to the problem. Figures 7 and 8 show the connected utility grid power purchase/sell (indicated by Taipower), DER generation and battery output with and without optimization, respectively. Table 1 indicates that optimal operation cost can be achieved by selling and purchasing power to/from the connected utility at different time steps based on the forecasted input. The cost saving without optimization is about $13 \%$ more than that with optimization.

\subsection{Case 2: Islanding Mode}

In this case, the load forecasting curve is shown in Figure 9 and the generation forecasts of uncontrollable DERs are the same those of Case 1. Figures $\mathbf{1 0}$ and $\mathbf{1 1}$ show the connected utility grid power purchase/sell (indicated by Taipower), DER generation and battery output with and without optimization, respectively. By observing Table 2, the cost saving with optimization is much less than that of Case 1. This is because that the islanding operation is without power purchase and sold to the utility grid.

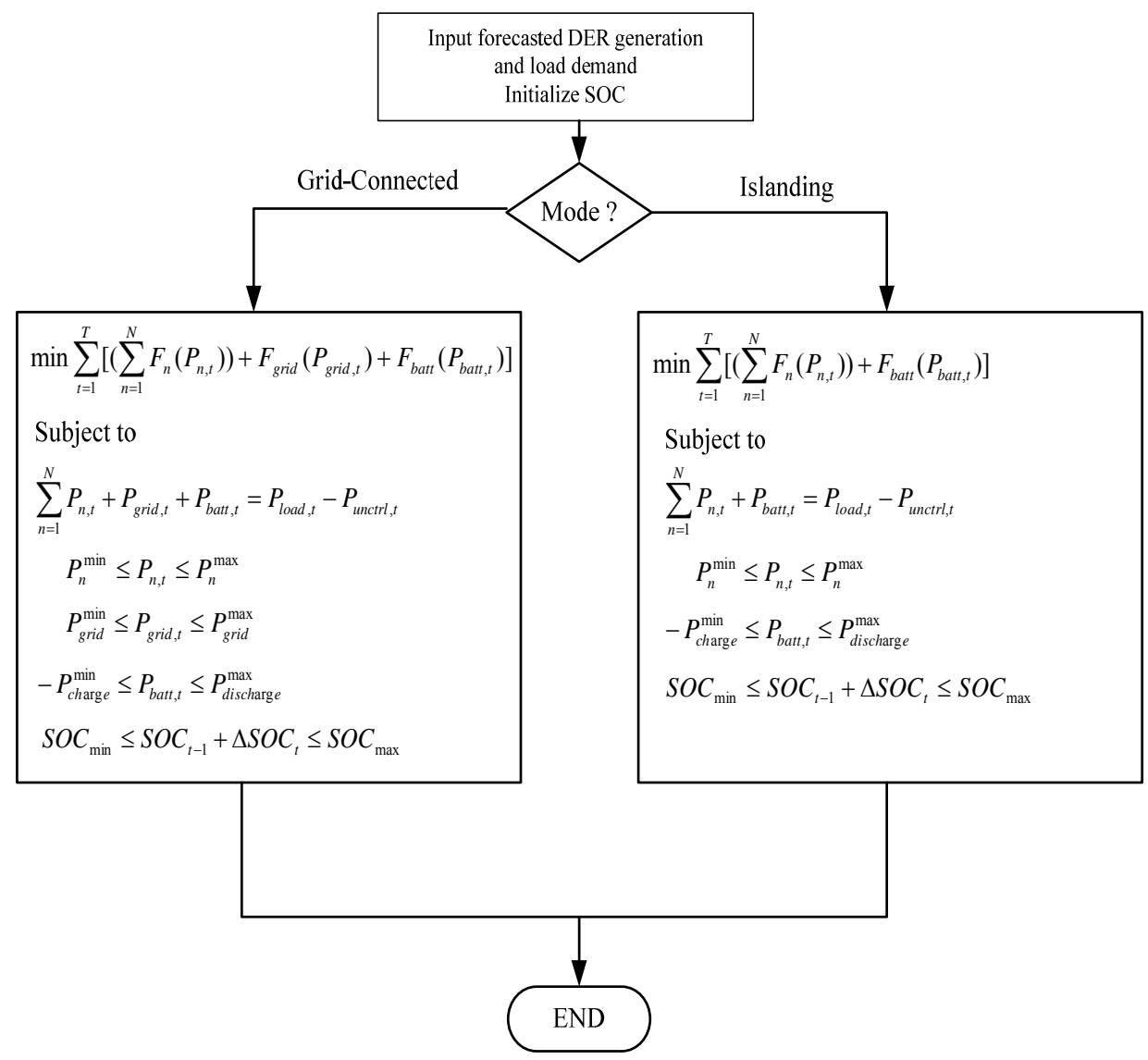

Figure 5. Flowchart of scheduling optimization program for grid-connected and islanding operations of the dc microgrid.

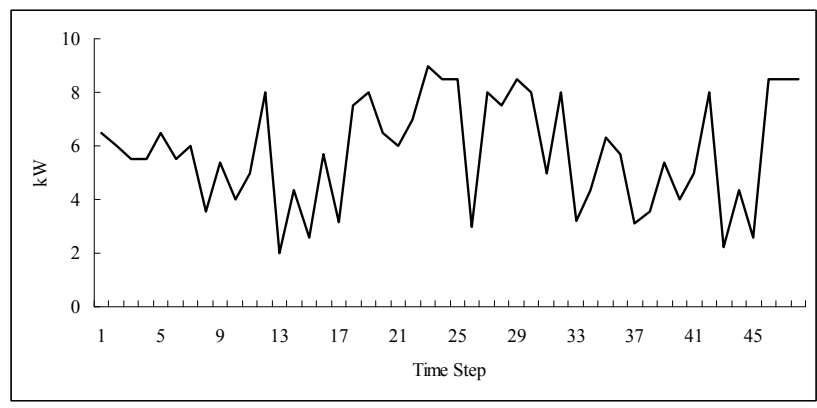

(a)

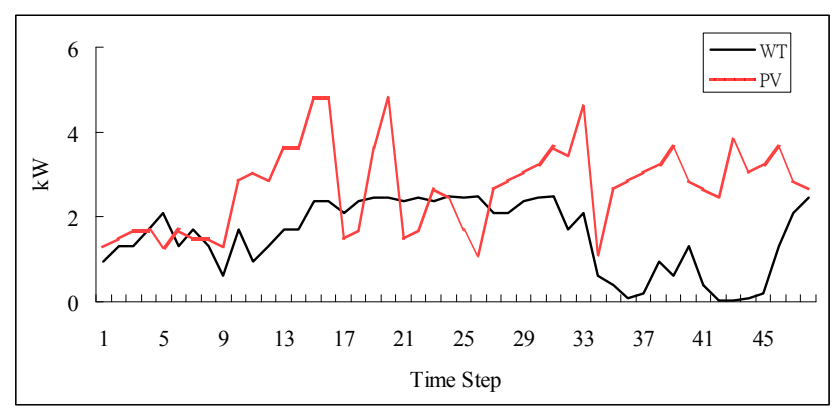

(b)

Figure 6. Forecasts of (a) load demand, and (b) uncontrollable DERs. 


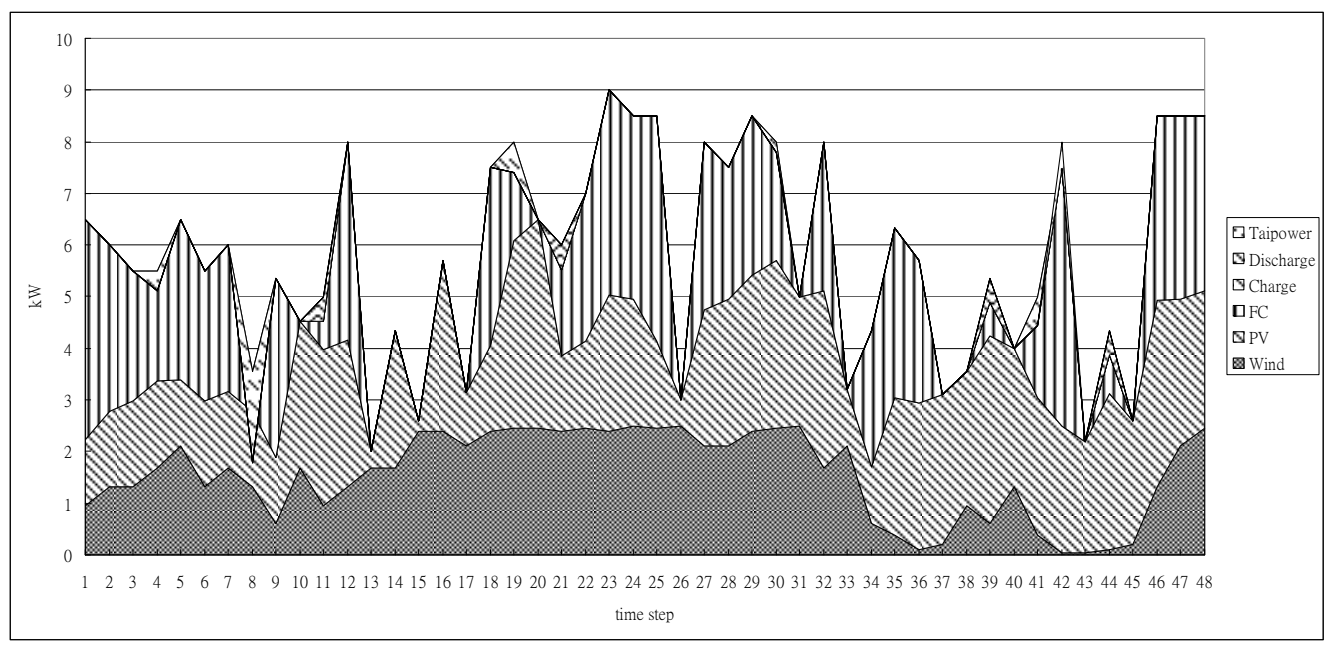

Figure 7. Power purchase/sell (Taipower), DER generations and battery output with optimization (Case 1).

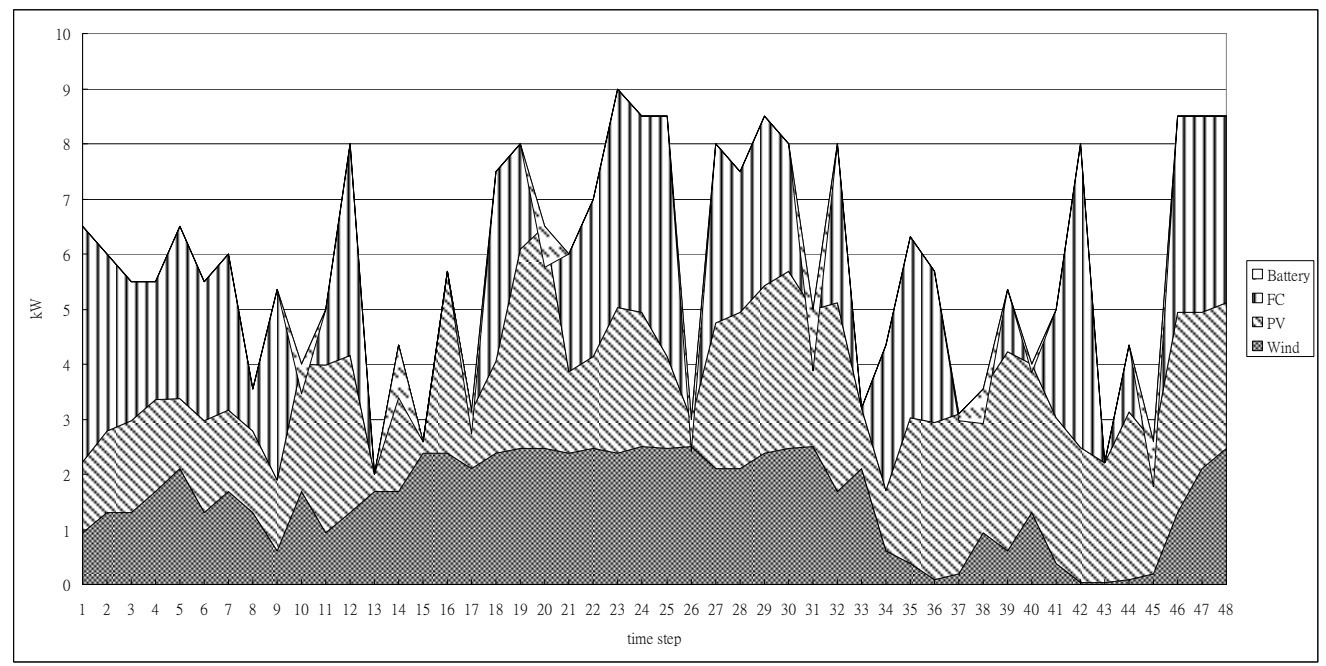

Figure 8. DER generations and battery output without optimization (Case 1).

Table 1. Comparison of operation cost (\$) w/o and with optimization.

\begin{tabular}{cccccc}
\hline & Fuel Cell & Battery & Sell & Purchase & Cost \\
\hline w/o optimization & 706.50 & 30.94 & 0.00 & 0.00 & 737.43 \\
With optimization & 694.00 & 8.33 & 60.33 & 1.55 & 642.00 \\
\hline
\end{tabular}

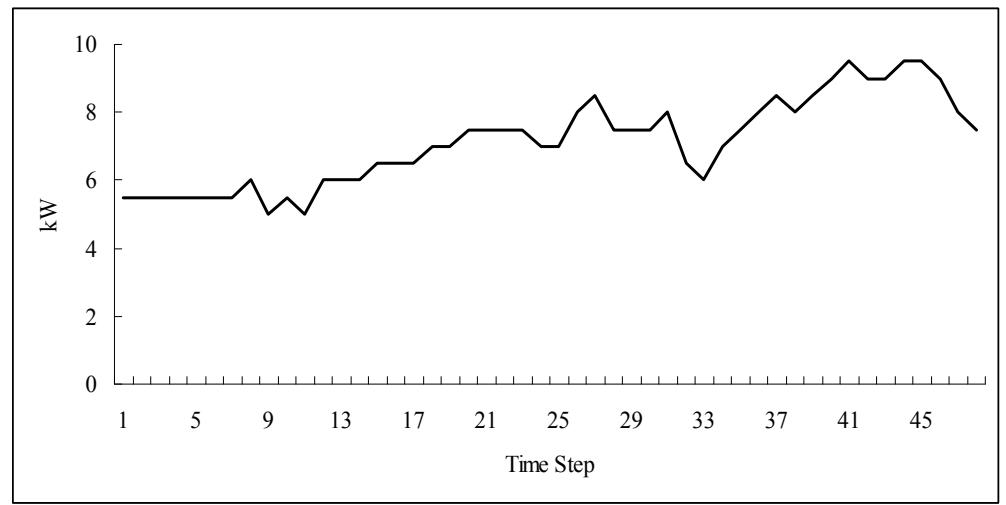

Figure 9. Load forecast for case 2. 


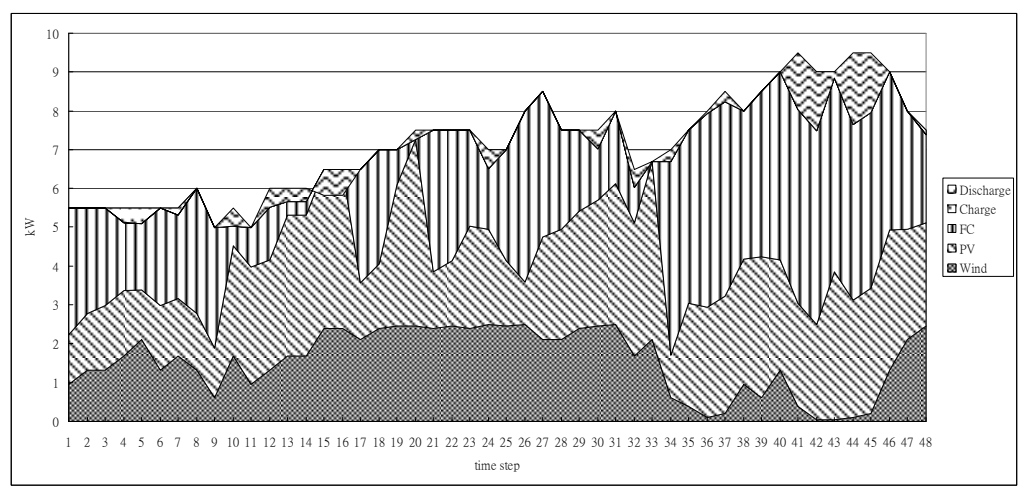

Figure 10. DER generations and battery output with optimization (Case 2).

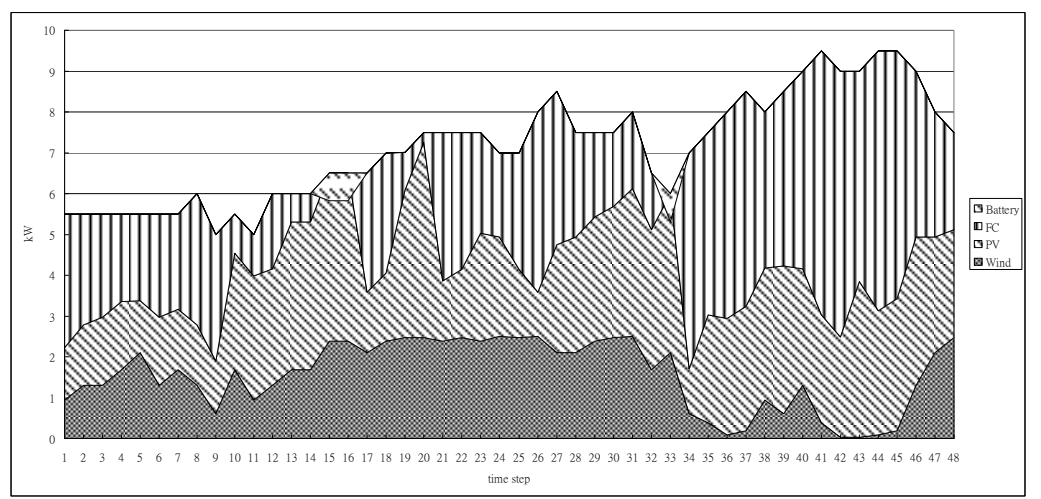

Figure 11. DER generations and battery output without optimization (Case 2).

Table 2. Comparison of operation Cost (\$) with and w/o Optimization

\begin{tabular}{llll}
\hline & Fuel Cell & Battery & Cost \\
\hline w/o optimization & 663.88 & 10.96 & 674.84 \\
With optimization & 641.43 & 24.41 & 665.84 \\
\hline
\end{tabular}

\section{Conclusions}

This paper has presented the aspect of short-term scheduling of DERs for an actual dc microgrid operated in both grid-connected and islanding modes. Results of two study cases were illustrated the usefulness of the proposed solution procedure to minimize the microgrid operation costs while satisfying all operation constraints. The schedules results were then input the time-domain simulation model to ensure a stable operation of microgrid with maintaining near fixed dc bus voltage under both grid-connected and islanding operations.

\section{REFERENCES}

[1] A. Niinistö, "Simulation of the Management of a MicroGrid with Wind, Solar and Gas Generators," Master's Thesis, Aalto University School of Science and Technology Faculty of Electronics, Finland, 2009.

[2] X. Liu, M. Ding, J. Han, P. Han and Y. Peng, "Dynamic
Economic Dispatch for Microgrids Including Battery Energy Storage," 2nd IEEE International Symposium on Power Electronics for Distributed Generation Systems (PEDG), 2010.

[3] F. Carastro, M. Sumner and P. Zanchetta, "An Enhancedshunt Active Filter with Energy Storage for Microgrids," Proceedings of IEEE Industry Applications Society Annual Meeting, Edmonton, October 2008.

[4] Y. Q. Zhu, Z. D. Yin and J. Tian, "Microgrids Based on DC Energy Pool," Proceedings of IEEE Energy 2030 Conference, Atlanta, November 2008. doi:10.1109/ENERGY.2008.4781004

[5] P. P. Dash and A. Yazdami, "A Mathematical Model and Performance Evaluation for a Single-stage Grid-connected Photovoltaic (PV) System," International Journal of Emerging Electric Power Systems, Vol. 9, No. 6, article 5, 2008.

[6] C. C. Yao, "Model Development and Simulation Analysis 
of Three-Phase Grid-Connected Photovoltaic Systems," Master's Thesis, Department Electric Engineering, National Taiwan University, Taipei, Taiwan, 2010.

[7] M. G. Villalva, J. R. Gazoli and R. Filho, "Comprehensive Approach to Modeling and Simulation of Photovoltaic Arrays," IEEE Transactions on Power Electronics, Vol. 24, No. 5, 2009, pp. 1198-1208.
doi:10.1109/TPEL.2009.2013862

[8] F. A Mohamed, "Microgrid Modeling and Online Management, Ph.D. Dissertation, Department of Automation and Systems Technology," Helsinki University of Technology Espoo, Finland, 2008.

[9] "SimPowerSystems Toolbox Users' Manual," Version 5.2, MathWorks Corp., 2010. 